\title{
NIHRAC approves six gene-transfer protocols
}

WASHINGTON, D.C.-At its March meeting, the National Institutes of Health Recombinant DNA Advisory Committee (NIHRAC, Bethesda, MD) approved six clinical gene-transfer protocols, while deferring four such protocols. In deciding those cases, however, committee members did little to dispel a sense that they won't move away from a purely case-by-case analysis of clinical gene-transfer proposals anytime soon, even though the issue could be considered at their next meeting, scheduled for June.

The NIHRAC meeting made clear that gene therapy's high profile is making some investigators squirm. For example, in outlining plans for gene-transfer tests on patients with life-threatening brain tumors, Kenneth Culver of the lowa Methodist Medical Center (Des Moines, IA) promised to report any progress to the committee. However, he urges NIHRAC not to transmit his interim reports onto "the front page of the newspapers. The publicity on compassionate use is making it more difficult for us." Indeed, recent sessions of NIHRAC have focused on compassionate-use requests and attendant efforts to forge policies concerning such requests.

\section{Four deferrals}

Difficulties aside, NIHRAC recommended approving Culver's plans, which were done in collaboration with John Van Gilder at the University of Iowa (Iowa City, IA), to introduce thymidine kinase genes from a herpes virus into brain tumor cells to render them more sensitive to the herpes drug ganciclovir. Despite safety concerns raised because the protocol uses previously untried genetic constructs and cell-packaging lines, the committee concluded that the deadly nature of the patients' malignancies outweighs the procedure's risks.

The committee also recommended approving a proposal from Jonathan Simons of the Johns Hopkins Oncology Center (Baltimore, MD) for treating patients with advanced kidney-cell cancer. Simons and his collaborators, in- cluding a group at Somatix Therapy (Alameda, CA), plan to introduce the gene for the cytokine, granulocyte-macrophage colony stimulating factor (GM-CSF), into cells of such patients. In studies of mice, gene-transfer-introduced GM-CSF helped to induce a strong antitumor response by the immune system, according to Simons. As a safety measure in the clinical study, plans call for irradiating cancer cells that are removed from a patient and engineered to contain the GM-CSF gene before they are reintroduced. As with the Culver protocol, the committee concluded that the potential benefits of testing this procedure on a limited number of patients outweigh the risks.

However, the committee reached opposite conclusions after considering four other proposals involving cancer patients. Among the proposals deferred:

-introducing multi-drug-resistance genes into cells from breast-cancer patients, by Arthur Bank of Columbia University (New York, NY);

-introducing the gamma-interferon gene into patients with malignant melanoma, by Hilliard Seigler of Duke University Medical Center (Durham, NC);

-introducing the interleukin-2 gene into patients with small-cell lung cancers, by Peter Cassileth and Eckhard Podack of the University of Miami (Miami, FL);

-and introducing drug-resistance genes into stem cells of patients under treatment for ovarian cancer, by Albert Deisseroth of the MD Anderson Cancer Center at the University of Texas (Houston, TX).

\section{Cystic flbrosis}

NIHRAC members are increasingly aware of a need for consistency, particularly when rejecting proposals. However, efforts to define explicit rejection criteria usually disappear in the specific objections committee members tend to frame for each proposal they defer. Nonetheless, models for success are emerging. For example, the committee considered and rapidly recommended for approval two proposals from Malcolm Brenner of St. Judes Children's Research Hospital (Memphis, TN) for introducing ge- netic markers into cancer patients undergoing bone-marrow-transplantation procedures. A veteran before NIHRAC, Brenner won praise for the polish of his proposals and for the way in which he justifies how many patients will be needed to complete the study.

Other proposals that were recommended for approval included two for treating individuals with cystic fibrosis $(\mathrm{CF})$, bringing the total to five CF protocols that have been approved since last December. The two recent proposals came from Richard Boucher and Michael Knowles of the University of North Carolina (Chapel Hill, NC) and from Robert Wilmott and Jeffrey Whitsett of Children's Hospital Medical Center (Cincinnati, OH), in collaboration with Bruce Trapnell of Genetic Therapy (Gaithersburg, MD).

Although NIHRAC typically restricts its concerns to those of safety and quality when evaluating proposals, broader viewpoints often emerge. Thus several committee members, now having okayed a handful of separate but similar CF gene-transfer procedures, are raising questions about the overlap and potential redundancy of these efforts. As committee member Gary Chase of the Johns Hopkins University (Baltimore, MD) puts it, this particular campaign in the "war on disease" may be growing "slightly wasteful." Several committee members thus urge the independent CF research groups to cooperate and share information. Yet some members discount the overlap, arguing that concentrated efforts on relatively rare genetic diseases such as CF need all the encouragement they can get.

The committee's policy regarding $\mathrm{CF}$ could grow ticklish as the CF commercial stakes are raised ever higher. For example, since receiving NIHRAC's initial approval of a CF protocol, Ronald Crystal, formerly of $\mathrm{NIH}$, has become part of a group that is forming a new company, GenVec (Montgomery County, MD), to do gene-therapyrelated research, including projects related to $\mathrm{CF}$. Among others, Genentech (S. San Francisco, CA) is an investor in GenVec.

-Jefirey L. Fox
NIHRAC has

done little to

dispel a sense

that it won't

move away

from a purely

case-by-case

analysis of

gene-transfer

proposals. 